\title{
Monitoring early stage lung disease in cystic fibrosis
}

Nissenbaum $C^{1.2}$, Davies $\mathrm{G}^{3,4}$, Horsley $\mathrm{A}^{1,2}$ Davies JC*5,6

1. Division of Infection, Immunity \& Respiratory Medicine, Manchester University, UK

2. Manchester Adult Cystic Fibrosis Centre, Manchester University Hospitals NHS Foundation Trust, UK

3. Great Ormond Street Institute of Child Health, University College London, UK

4. Great Ormond Street Hospital, London, UK

5. National Heart and Lung Institute, Imperial College London, UK

6. The Royal Brompton \& Harefield NHS Foundation Trust, London, UK

\section{Contact details}

Professor Jane Davies (*corresponding author)

E-mail: j.c.davies@imperial.ac.uk Tel: 02075947973

Dr Gwyneth Davies

E-mail: gwyneth.davies@ucl.ac.uk Tel:020 76792000

Dr Alexander Horsley

E-mail: alexander.horsley@manchester.ac.uk Tel: 01613066000

Dr Claire Nissenbaum

E-mail: claire.nissenbaum@manchester.ac.uk Tel: 01613066000 


\section{Abstract}

Purpose

Early stage lung disease has long been synonymous with infancy and childhood. As diagnosis happens earlier and conventional management improves, we are seeing larger proportions of people with CF in adolescence and even adulthood with well-preserved lung health. The availability of highly effective CFTR modulator drugs for a large proportion of the CF population will impact even further. Transitioning into adult care with 'normal' lung function will become more common.

However, it is crucial that we are not blasé about this phase, which sets the scene for future lung health. It is well-recognised that lung function assessed by spirometry is insensitive to 'early' changes occurring in the distal, small airways. Much of our learning has come from studies in infants and young children, which have allowed assessment and optimisation of alternative forms of monitoring.

\section{Recent findings}

Here, as a group of paediatric and adult CF specialists, we review the evidence base for sensitive physiological testing based on multibreath washout, lung imaging, exercise and activity monitoring, assessment of infection and quality of life measures.

\section{Summary}

We seek to emphasise the importance of further work in these areas, as outcome measures become widely applicable to a growing CF population.

Keywords Cystic fibrosis, Lung clearance index, Computerised Tomography, Magnetic resonance imaging, Outcome measures 


\section{Introduction}

The term 'early stage' lung disease in cystic fibrosis (CF) has long been synonymous with 'early life', i.e. infancy and childhood. However, as diagnosis is increasingly made with newborn screening (NBS) and as both conventional and mutation-based therapies advance, this term will become relevant also to increasing numbers of older people with (pw)CF. In our opinion, the use of the term 'mild' is inappropriate and may lead to complacency and under-treatment; many early stage changes are not insubstantial. They are, however, more challenging to detect. Sensitive methods to assess early stage lung disease are required to track clinical progression, guide management and detect efficacy (or harm) in clinical trials. Here, we describe current clinical challenges and highlight some of the paths forward.

\section{Predicted impacts of highly effective CFTR modulators.}

The last decade has witnessed a paradigm shift in treatment for CF. Whereas previously, all therapies targeted the consequences of CFTR dysfunction (mucus composition and accumulation, infection), CFTR modulators target the CFTR protein itself, restoring function as an ion channel (1). Ivacaftor has demonstrated substantial durability (2), with a slowing in loss of lung function, fewer exacerbations, improvements in nutrition and quality of life. Most accept that even complete restoration of CFTR function will not reverse established, structural lung damage. However, commencement at an earlymoderate stage may substantially slow the development of lung disease, or even prevent its onset. Indeed, restoration of pancreatic exocrine function, previously considered irreversible, has been now been seen in children starting ivacaftor (3-5). As we write, the European Medicines Agency (EMA) has approved the latest such drug (elexacaftor/ tezacaftor/ ivacaftor; Kaftrio). The acute impacts of this combination, in pwCF possessing either 1 (6) or 2 (7) copies of the F508del mutation ( $85 \%$ of global population) are substantial, at least as good or exceeding those with ivacaftor. Trials in 6-11 year old children are underway (Clinicaltrials.gov NCT04043806) and are planned for younger age groups. The 
widespread uptake of these highly effective modulator therapies (HEMT) will change the face of CF dramatically.

\section{What we have learned from disease monitoring in early life}

Most infants and many children with CF are asymptomatic from the chest point of view. Detection of lung disease in this group poses several challenges: the requirement for highly sensitive tests, the child's small size and their inability/ unwillingness to co-operate. Conventional spirometry is not possible early in life. Specialist labs have developed techniques to measure volumes/flows, although they are largely applied on a research basis. They include the raised volume rapid thoracoabdominal compression technique (RVRTC), plethysmography, and multiple breath washout (MBW, see later). Two key research collaborations have monitored the development of CF lung disease from infancy: the Australian Respiratory Early Surveillance Team for Cystic Fibrosis (AREST-CF), and the London Cystic Fibrosis Collaboration (LCFC)(8-12). LCFC infants diagnosed by NBS had abnormal FEV 0.5 at 3 months of age (9), which improved over the first year (10). Aged 2, there was no significant difference between healthy controls and CF (13). This contrasts with a dramatic decline in infant lung function over this period in AREST-CF $(8,11)$. Possible explanations for this difference remain to be resolved (14).

Both groups have evaluated the role of chest CT in early CF life. Aged 1 year, LCFC NBS infants showed only mild abnormalities on the CF-CT Brody II system $(15,16)$. This may reflect true minimal lung disease or an insensitive scoring system. The Perth-Rotterdam Annotated Grid Morphometric Analysis for CF (PRAGMA-CF) scoring system was developed to detect the mildest abnormalities on chest CT and be more discriminative than CF-CT (17). AREST-CF reported a $20 \%$ prevalence for bronchiectasis and $58 \%$ for air trapping at 1 year of age in NBS infants (18), but the mean extent of bronchiectasis was only $0.2 \%$ of the lung. Novel methods of quantifying structural lung abnormalities (such as counting airway segments (19)), or automated approaches to quantitative measurement of air 
trapping will further help to elucidate the relationship between structure and function outcomes in CF and will be applicable at all ages.

Detection of neutrophilic inflammation is a prognostic marker for lung disease in the early years. AREST-CF reported the association between free neutrophil elastase (NE) activity at 3 months and bronchiectasis from one year of age (20). Repeated detection of free NE is associated with worse CT outcomes (21). They also reported an association between detectable NE in bronchoalveolar lavage (BAL) at 3 months of age and isolation of Staphylococcus aureus and Pseudomonas aeruginosa, and significantly lower lung function during the first 2 years of life (11). In the NBS LCFC cohort, inflammation and current/ prior infection was associated with only mild abnormalities on chest CT and lung function at 1 year of age (22). Ongoing follow up of these cohorts will determine the importance of these outcomes in later childhood and adolescence.

\section{Sensitive physiology testing: MBW and Lung Clearance Index}

MBW tests have emerged over the last decade as a highly sensitive method of identifying early changes in obstructive airways diseases, and particularly in CF (23). MBW involves tidal breathing to washout an inert tracer gas, typically either exogenous sulphur hexafluoride $\left(\mathrm{SF}_{6}\right)$ or resident nitrogen, the latter washed out by $100 \%$ oxygen. The washout starts after a relaxed expiration, with the lung at functional residual capacity (FRC). The simplest and most commonly used derived measure is lung clearance index $(\mathrm{LCl})(24)$, the total breath volume required to wash the inert gas out to $1 / 40^{\text {th }}$ of its starting concentration, expressed as multiples of the FRC. Lung disease in CF is patchy and uneven; more diseased lung regions are less well ventilated and wash tracer gas out more slowly. A high $\mathrm{LCl}$ is thus a marker of more significant lung disease.

LCl sensitively detects early CF airway abnormalities from infants to adults (25) even those with normal spirometry (26-31). It can be measured in very young children with high rates of success (32). It is possible in infants, though requires different approaches $(27,33,34) . \mathrm{LCl}$ tracks $\mathrm{CF}$ lung disease more 
sensitively than spirometry, changing with inflammation and improving with treatment (35-40). Significant pulmonary infection is associated with increased $\operatorname{LCI}(25,38,41-43)$ and an accelerated rate of progression $(43,44)$. For disease monitoring, a strength of $\mathrm{LCl}$ is that the upper limit of normal remains very stable, including during adolescence (45-47) facilitating assessment of longitudinal change (48). In stable preschool and school age children with CF, LCl has a repeatability of $+/-25 \%$ baseline LCI (49-51), worse than in healthy subjects or in adults with CF, but comparable to spirometry (52).

The challenges of measuring lung function in infants described above make LCl well-suited to this population. The AREST-CF cohort showed normal or impaired LCI in the first 6 months of life with deterioration thereafter in children with a history of pulmonary infections (43). The London group reported that NBS infants with increased or normal LCl tracked to one (10), but not to two, years of age. $(13,44)$. The LCFC have recently reported the association between preschool and adolescent LCI in a clinically diagnosed cohort $(10,53)$ Longer term follow-up of these cohorts will help inform the predictive value of very early assessments in NBS populations.

There are, as with all physiological measurements, some important challenges in measuring LCI. In infants sedation is usually required $(43,54)$. In younger children, success is the norm above around 3 years old, but requires a controlled environment and an experienced team (32). Testing takes longer than spirometry, placing limitations on scale up to routine clinical use $(55,56)$.

In clinical trials recruiting children with well-preserved baseline $\mathrm{FEV}_{1}, \mathrm{LCl}$ has allowed demonstration of efficacy; examples include RhDNase, dual-combination CFTR modulator therapies and nebulised hypertonic saline (57-62). Procedures have been standardised across CF trial networks globally for one MBW technique, which is being increasingly adopted into paediatric (63) and adult studies. LCI may also possess sufficient sensitivity to be used in future trials of additional therapies once HEMT is standard of care and incremental improvements are anticipated to be small.

Inversely, $\mathrm{LCl}$ is less reliable in more severe disease or pulmonary exacerbations $(36,37)$. In more advanced disease, duration of tests can also be unacceptably long. There are also important 
differences between measurement systems and tracer gases; results are not comparable between different technologies $(56,64,65)$.

\section{Exercise testing/ activity monitoring}

Maximal exercise capacity $\left(\mathrm{VO}_{2} \max \right)$ is associated with $\mathrm{CF}$ mortality (66-68). Current evidence supports a role in CF in providing prognostic information (death or transplant); its role in early stage CF lung disease is less well established. Furthermore, although recommended as part of standard care from 10 years of age $(69,70)$, it is not performed in all CF centres. There are limitations e.g. younger participants may not have sufficient leg length or achieve appropriate cadence. Field exercise tests, (6 minute walk (71) and shuttle (72)), may be sub-maximal in health and in mild CF lung disease: the 15level Modified Shuttle Test (MST) is sub-maximal in $30 \%$ of healthy individuals and $6 \%$ of adults with CF (73). Further modifications have been developed to address this (73).

Activity monitoring quantifies physical activity undertaken in daily life with questionnaires or, objectively, with wearable sensors. Self-reported physical activity is associated with rate of decline in $\mathrm{FEV}_{1}$ in $\mathrm{CF}$ : those reporting an increase in habitual physical activity over a nine year period had slower lung function loss (74). Similar levels of physical activity have been reported between children/adults with CF and healthy controls, except at very low lung function when those with CF may be less active (75). However, there may be differences in the intensity of activity being undertaken, with school aged children with CF spending less time undertaking strenuous or vigorous activities than controls $(75,76)$. Other groups have detected a CF-health difference in activity even in mild disease (77).

\section{Advances in Lung imaging}

One of the most powerful emerging technologies to measure and track early CF lung disease is magnetic resonance imaging (MRI). Conventional MRI detects protons in lung tissue reading out structure similarly to HRCT. Advanced MR techniques provide high resolution images rivalling CT (78), 
and imaging is possible to sixth generation bronchi (79). Neither CT nor MRI can image smaller airways, early disease more distally being identified instead by mucus plugging and gas trapping. The main advantage of structural MRI over CT is radiation-free imaging $(80,81)$. Multi-centre trials have demonstrated the feasibility of repeated scans $(25,61)$, and standardised scoring systems are available (82). Numerous additional MR imaging techniques also exist to enhance the data available from proton imaging, or to make use of injectable contrast agents (83). The unique power of MRI however lies in the ability to introduce inhaled contrast (83), such as hyperpolarised helium $\left(\mathrm{He}^{3}\right)$ or xenon $\left(\mathrm{Xe}^{129}\right)$. These provide detailed 3-dimensional representations of breath distribution and can pick out regions affected by airway blockage below the resolution of structural imaging by CT or MRI. $\mathrm{He}^{3}-\mathrm{MRI}$ has been shown to be the most sensitive measure of airway obstruction, being abnormal in those with well-preserved spirometry and normal $\mathrm{LCl}(80)$. It has also shown disease progression over time despite stable spirometry and stable LCI (31). The detection of ventilation defects however does not tell us specifically what the cause is. For those with normal spirometry, the appearances are typically of multiple small ventilation defects which disappear on deep-breath imaging (84). This suggests small airway obstruction, for instance caused by mucus, which may be amenable to intervention. In those with abnormal $\mathrm{FEV}_{1}$ however, $\geq 10 \%$ of lung volume is obstructed during tidal breathing, and less reversibility is seen on deep inhalation (84), suggesting a fixed obstruction. Ivacaftor resulted in very marked improvement in ventilation imaging in adults with the G551D mutation (85).

$\mathrm{He}^{3}$ provides the highest quality ventilation-MR images, but its expense has led to increasing use of $\mathrm{Xe}^{129}$, more widely available and cheaper but possessing a poorer signal-to-noise ratio and potential for short-lived systemic absorption (86). Xenon appears to provide similar information to Helium imaging, is repeatable and able to detect changes over time and in response to intervention $(87,88)$. It is likely that its practical advantages will lead to Xe being the standard for future long-term studies and clinical trials involving ventilation-MRI.

Hyperpolarised gas-free techniques have developed in parallel and provide both functional and structural information. Oxygen-enhanced MRI is a potentially attractive example, providing a 
composite measure of alveolar ventilation, diffusion and perfusion. One study has reported its correlation with spirometry and CT scores in pwCF (89) and a larger studies are ongoing. MRI remains more technically complex and expensive than lung function measurements like $\mathrm{LCl}$, requires sedation for young children, and has only been proposed as a routine annual assessment in highly specialist centres (90). In the short term it is likely to remain restricted to specialist use but could help considerably in the assessment of interventions early in disease progression.

\section{Challenges with infection surveillance}

Real-time awareness of an individual's lower airway infection status allows prompt intervention which may improve long-term outcomes. This is relatively easy in patients with moderate-severe lung disease, most of whom chronically producing sputum. Although sputum culture 'misses' many organisms detectable on molecular analysis (91), the clinical importance of these remains unclear. Most children and an increasing number of adult pwCF do not produce sputum and this will become more common once highly effective CFTR modulators are widely available. Surveillance in this instance relies routinely on less sensitive/ specific 'cough' or throat swabs (92). Induction of sputum with hypertonic saline nebulisation is useful but time-consuming and therefore not frequently repeatable (93). Bronchoalveolar lavage, the 'gold-standard' is invasive, requires general anaesthesia and is largely used when other measures to obtain secretions in a child causing clinical concern have failed. This is an area of unmet clinical need requiring new techniques, likely advanced technology based, to be resolved.

\section{Assessing Quality of Life and psychological health}

The CFQ- $R$ is a disease-specific quality of life(QoL) questionnaire, with domains including respiratory symptoms, treatment burden and physical functioning (94); higher scores (better QoL) are associated with less severe lung disease assessed according to $\mathrm{FEV}_{1}(94)$. Different versions are available: $14 \mathrm{yrs}+$ (CFQ Teen/adult), 6-13 years, and parent. A modified version for parents of children $<6$ years was 
utilised in the Infant Study of Inhaled Saline clinical trial $(95,96)$. Perrem et al have recently demonstrated the discriminant validity of the parental CFQ-R Respiratory Symptoms domain score which deteriorates with acute respiratory events in preschool children and improves with treatment (97). The CFQ-R respiratory domain has been used as an outcome in clinical trials including those involving mild lung disease. A clinical trial of ivacaftor in those with preserved spirometry $(>90 \%$ predicted $\mathrm{FEV}_{1}$ ) met its primary endpoint of significant improvement in $\mathrm{LCl}$ but did not detect a significant difference in the change in the CFQ-R respiratory domain score between treatment and placebo groups (98). It is likely that this tool is insufficiently sensitive in early stage disease and alternatives are required.

\section{Looking to the future}

The population with CF is growing as survival improves. Modern care, including early diagnosis with NBS has incrementally improved lung health and the widespread use of highly effective modulator drugs will impact this hugely. Never has there been a more pressing need for tools to monitor disease in patients at the earlier stages of CF severity. Drugs will continue to be needed, but the testing of these will become more complex if being applied on top of HEMT (99). Measures such as those discussed above will be needed as trial outcomes and require standardisation and a robust evidence base.

In the second quarter of 2020 , the CF clinical community, our patients and their families adapted rapidly to the acute health threat of the global covid-19 pandemic. Home monitoring, to date an area of pilot research and feasibility studies, became a necessity, with video-consultations, hand-held spirometers and weighing scale provision becoming widespread. The impact of these essential measures on data quality, longer term outcomes and emotional well-being requires assessment, but it seems likely that we will use the experience gained to establish new ways of working once the heat of the pandemic has cooled. Personalised care, particularly as population health improves with HEMT, is likely to involve fewer in-person clinic visits and a reduced need for hospitalisation. We urgently 
need to establish a robust infrastructure with appropriately trained teams, suitable IT capacity and information governance structures to deliver this. Advances in technology including wearables, adherence aids and point-of-care and breath-based infection diagnostics are encouraging. Implementation of any of these needs to decrease rather than add to the burden of care for pwCF $(100,101)$ and mandates assessment in partnership with the patient community.

\section{Conclusions}

The health of people with CF has been improving over recent decades. The introduction of highly effective CFTR modulator drugs will have further beneficial impact. Whilst this is hugely encouraging, it does present some challenges with regard to tracking lung disease. The absence of a measurable defect in spirometry does not indicate the absence of inflammation, infection and remodelling. Paediatric respiratory medicine has grappled with the requirement for more sensitive measuring tools for many years, providing much experience upon which we can build. An evidence base is building for lung clearance index and progress is also being made with imaging modalities; those free of radiation, whilst some way behind $\mathrm{CT}$, may enable frequent assessment. Further work is needed in these, and other areas. In order that these assessments in themselves do not add to the burden of management already experienced by people with $\mathrm{CF}$, we advocate a partnership approach with patient representative organisations and lay experts.

\section{Key points}

- Early stage lung disease is increasingly present in adolescents and adults with CF and will become more common with the advent of highly effective modulator therapy for a majority of patients

- Conventional spirometry is unhelpful in monitoring this stage of disease, mandating the use of more sensitive tools 
- Experience built in paediatric centres with sensitive physiology testing and imaging is providing a useful foundation for optimisation of newer approaches to lung disease monitoring

\section{Acknowledgement}

$\mathrm{CN}$ is supported by grants from the CF Foundation and from the North West Lung Centre charity. AH is supported by NIHR Manchester Biomedical Research Centre. GD is supported by the NIHR Great Ormond Street Hospital Biomedical Research Centre. JCD is supported by the NIHR through a Senior Investigator Award, the Imperial Biomedical Research Centre and the Royal Brompton Hospital/ Imperial College London Clinical Research Facility. The views expressed as those of the authors and not necessarily those of the NHS, the NIHR or the Department of Health.

\section{References}

1. Joshi D, Ehrhardt A, Hong JS, Sorscher EJ. Cystic fibrosis precision therapeutics: Emerging considerations. Pediatric pulmonology. 2019;54 Suppl 3:S13-S7.

2. Volkova N, Moy K, Evans J, Campbell D, Tian S, Simard C, et al. Disease progression in patients with cystic fibrosis treated with ivacaftor: Data from national US and UK registries. Journal of cystic fibrosis : official journal of the European Cystic Fibrosis Society. 2020;19(1):68-79.

3. Davies JC, Cunningham S, Harris WT, Lapey A, Regelmann WE, Sawicki GS, et al. Safety, pharmacokinetics, and pharmacodynamics of ivacaftor in patients aged 2-5 years with cystic fibrosis and a CFTR gating mutation (KIWI): an open-label, single-arm study. The lancet Respiratory medicine. 2016;4(2):107-15.

4. Rosenfeld M, Cunningham S, Harris WT, Lapey A, Regelmann WE, Sawicki GS, et al. An openlabel extension study of ivacaftor in children with CF and a CFTR gating mutation initiating treatment at age 2-5years (KLIMB). Journal of cystic fibrosis : official journal of the European Cystic Fibrosis Society. 2019;18(6):838-43.

5. Nichols AL, Davies JC, Jones D, Carr SB. Restoration of exocrine pancreatic function in older children with cystic fibrosis on ivacaftor. Paediatric respiratory reviews. 2020.

6. Middleton PG, Mall MA, Drevinek P, Lands LC, McKone EF, Polineni D, et al. ElexacaftorTezacaftor-Ivacaftor for Cystic Fibrosis with a Single Phe508del Allele. The New England journal of medicine. 2019;381(19):1809-19.

7. Heijerman HGM, McKone EF, Downey DG, Van Braeckel E, Rowe SM, Tullis E, et al. Efficacy and safety of the elexacaftor plus tezacaftor plus ivacaftor combination regimen in people with cystic 
fibrosis homozygous for the F508del mutation: a double-blind, randomised, phase 3 trial. Lancet. 2019;394(10212):1940-8.

8. Linnane BM, Hall GL, Nolan G, Brennan S, Stick SM, Sly PD, et al. Lung function in infants with cystic fibrosis diagnosed by newborn screening. American journal of respiratory and critical care medicine. 2008;178(12):1238-44.

9. Hoo AF, Thia LP, Nguyen TT, Bush A, Chudleigh J, Lum S, et al. Lung function is abnormal in 3month-old infants with cystic fibrosis diagnosed by newborn screening. Thorax. 2012;67(10):874-81.

10. Nguyen TT, Thia LP, Hoo AF, Bush A, Aurora P, Wade A, et al. Evolution of lung function during the first year of life in newborn screened cystic fibrosis infants. Thorax. 2014;69(10):910-7.

11. Pillarisetti N, Williamson E, Linnane B, Skoric B, Robertson CF, Robinson P, et al. Infection, inflammation, and lung function decline in infants with cystic fibrosis. American journal of respiratory and critical care medicine. 2011;184(1):75-81.

12. Ranganathan SC, Dezateux C, Bush A, Carr SB, Castle RA, Madge S, et al. Airway function in infants newly diagnosed with cystic fibrosis. Lancet. 2001;358(9297):1964-5.

13. Davies G, Stocks J, Thia LP, Hoo AF, Bush A, Aurora P, et al. Pulmonary function deficits in newborn screened infants with cystic fibrosis managed with standard UK care are mild and transient. The European respiratory journal. 2017;50(5).

14. Bush A, Sly PD. Evolution of cystic fibrosis lung function in the early years. Current opinion in pulmonary medicine. 2015;21(6):602-8.

15. Thia LP, Calder A, Stocks J, Bush A, Owens CM, Wallis C, et al. Is chest CT useful in newborn screened infants with cystic fibrosis at 1 year of age? Thorax. 2014;69(4):320-7.

16. Brody AS, Kosorok MR, Li Z, Broderick LS, Foster JL, Laxova A, et al. Reproducibility of a scoring system for computed tomography scanning in cystic fibrosis. Journal of thoracic imaging. 2006;21(1):14-21.

17. Rosenow T, Oudraad MC, Murray CP, Turkovic L, Kuo W, de Bruijne M, et al. PRAGMA-CF. A Quantitative Structural Lung Disease Computed Tomography Outcome in Young Children with Cystic Fibrosis. American journal of respiratory and critical care medicine. 2015;191(10):1158-65.

18. Ramsey KA, Rosenow T, Turkovic L, Skoric B, Banton G, Adams AM, et al. Lung Clearance Index and Structural Lung Disease on Computed Tomography in Early Cystic Fibrosis. American journal of respiratory and critical care medicine. 2016;193(1):60-7.

19. Verbanck S, King GG, Zhou W, Miller A, Thamrin C, Schuermans D, et al. The quantitative link of lung clearance index to bronchial segments affected by bronchiectasis. Thorax. 2018;73(1):82-4.

20. Sly PD, Gangell CL, Chen L, Ware RS, Ranganathan S, Mott LS, et al. Risk factors for bronchiectasis in children with cystic fibrosis. The New England journal of medicine. 2013;368(21):1963-70.

21. Rosenow T, Mok LC, Turkovic L, Berry L, Sly PD, Ranganathan S, et al. The cumulative effect of inflammation and infection on structural lung disease in early cystic fibrosis. The European respiratory journal. 2019;54(1).

22. Davies G, Thia LP, Stocks J, Bush A, Hoo AF, Wade A, et al. Minimal change in structural, functional and inflammatory markers of lung disease in newborn screened infants with cystic fibrosis at one year. Journal of cystic fibrosis : official journal of the European Cystic Fibrosis Society. 2020.

23. Horsley A, Siddiqui S. Putting lung function and physiology into perspective: cystic fibrosis in adults. Respirology. 2015;20(1):33-45.

24. Horsley A. Lung clearance index in the assessment of airways disease. Respiratory medicine. 2009;103(6):793-9.

25. Stahl M, Wielputz MO, Graeber SY, Joachim C, Sommerburg O, Kauczor HU, et al. Comparison of Lung Clearance Index and Magnetic Resonance Imaging for Assessment of Lung Disease in Children with Cystic Fibrosis. Am J Respir Crit Care Med. 2017;195(3):349-59.

26. Stahl M, Joachim C, Blessing K, Hammerling S, Sommerburg O, Latzin P, et al. Multiple breath washout is feasible in the clinical setting and detects abnormal lung function in infants and young children with cystic fibrosis. Respiration. 2014;87(5):357-63. 
27. Stahl M, Graeber SY, Joachim C, Barth S, Ricklefs I, Diekmann G, et al. Three-center feasibility of lung clearance index in infants and preschool children with cystic fibrosis and other lung diseases. Journal of cystic fibrosis : official journal of the European Cystic Fibrosis Society. 2018;17(2):249-55.

28. Horsley AR, Gustafsson PM, Macleod KA, Saunders C, Greening AP, Porteous DJ, et al. Lung clearance index is a sensitive, repeatable and practical measure of airways disease in adults with cystic fibrosis. Thorax. 2008;63(2):135-40.

29. Benseler A, Stanojevic S, Jensen R, Gustafsson P, Ratjen F. Effect of equipment dead space on multiple breath washout measures. Respirology. 2015;20(3):459-66.

30. Gustafsson PM. Inert gas washout in preschool children. Paediatric respiratory reviews. 2005;6(4):239-45.

31. Smith L, Marshall H, Aldag I, Horn F, Collier G, Hughes D, et al. Longitudinal Assessment of Children with Mild Cystic Fibrosis Using Hyperpolarized Gas Lung Magnetic Resonance Imaging and Lung Clearance Index. American journal of respiratory and critical care medicine. 2018;197(3):397400.

32. Downing B, Irving S, Bingham Y, Fleming L, Bush A, Saglani S. Feasibility of lung clearance index in a clinical setting in pre-school children. The European respiratory journal. 2016;48(4):1074-80.

33. Shawcross A, Murray CS, Pike K, Horsley A. A novel method for infant multiple breath washout: First report in clinical practice. Pediatric pulmonology. 2019;54(8):1284-90.

34. Kentgens AC, Guidi M, Korten I, Kohler L, Binggeli S, Singer F, et al. Infant multiple breath washout using a new commercially available device: Ready to replace the previous setup? Pediatric pulmonology. 2018;53(5):628-35.

35. Robinson PD, Goldman MD, Gustafsson PM. Inert gas washout: theoretical background and clinical utility in respiratory disease. Respiration. 2009;78(3):339-55.

36. Sonneveld N, Stanojevic S, Amin R, Aurora P, Davies J, Elborn JS, et al. Lung clearance index in cystic fibrosis subjects treated for pulmonary exacerbations. The European respiratory journal. 2015;46(4):1055-64.

37. Horsley AR, Davies JC, Gray RD, Macleod KA, Donovan J, Aziz ZA, et al. Changes in physiological, functional and structural markers of cystic fibrosis lung disease with treatment of a pulmonary exacerbation. Thorax. 2013;68(6):532-9.

38. O'Neill K, Bradley JM, Reid A, Downey DG, Rendall J, McCaughan J, et al. Airway infection, systemic inflammation and lung clearance index in children and adults with cystic fibrosis. The European respiratory journal. 2018;51(2).

39. Welsh L, Nesci C, Tran H, Tomai M, Ranganathan S. Lung clearance index during hospital admission in school-age children with cystic fibrosis. Journal of cystic fibrosis : official journal of the European Cystic Fibrosis Society. 2014;13(6):687-91.

40. Yammine S, Bigler A, Casaulta C, Singer F, Latzin P. Reasons for heterogeneous change in LCI in children with cystic fibrosis after antibiotic treatment. Thorax. 2014;69(2):183.

41. Belessis Y, Dixon B, Hawkins G, Pereira J, Peat J, MacDonald R, et al. Early cystic fibrosis lung disease detected by bronchoalveolar lavage and lung clearance index. American journal of respiratory and critical care medicine. 2012;185(8):862-73.

42. Aurora P, Gustafsson P, Bush A, Lindblad A, Oliver C, Wallis CE, et al. Multiple breath inert gas washout as a measure of ventilation distribution in children with cystic fibrosis. Thorax. 2004;59(12):1068-73.

43. Simpson SJ, Ranganathan S, Park J, Turkovic L, Robins-Browne RM, Skoric B, et al. Progressive ventilation inhomogeneity in infants with cystic fibrosis after pulmonary infection. The European respiratory journal. 2015;46(6):1680-90.

44. Ramsey K, Ratjen F, Latzin P. Elucidating progression of early cystic fibrosis lung disease. The European respiratory journal. 2017;50(5).

45. Horsley AR, Alrumuh A, Bianco B, Bayfield K, Tomlinson J, Jones A, et al. Lung clearance index in healthy volunteers, measured using a novel portable system with a closed circuit wash-in. PloS one. 2020;15(2):e0229300. 
46. Lum S, Stocks J, Stanojevic S, Wade A, Robinson P, Gustafsson P, et al. Age and height dependence of lung clearance index and functional residual capacity. The European respiratory journal. 2013;41(6):1371-7.

47. Anagnostopoulou $P$, Latzin $P$, Jensen $R$, Stahl M, Harper A, Yammine S, et al. Normative data for multiple breath washout outcomes in school-aged Caucasian children. The European respiratory journal. 2020;55(4).

48. Kraemer R, Blum A, Schibler A, Ammann RA, Gallati S. Ventilation inhomogeneities in relation to standard lung function in patients with cystic fibrosis. American journal of respiratory and critical care medicine. 2005;171(4):371-8.

49. Oude Engberink E, Ratjen F, Davis SD, Retsch-Bogart G, Amin R, Stanojevic S. Inter-test reproducibility of the lung clearance index measured by multiple breath washout. The European respiratory journal. 2017;50(4).

50. Stanojevic S, Davis SD, Retsch-Bogart G, Webster H, Davis M, Johnson RC, et al. Progression of Lung Disease in Preschool Patients with Cystic Fibrosis. American journal of respiratory and critical care medicine. 2017;195(9):1216-25.

51. Green K, Kongstad T, Skov M, Buchvald F, Rosthoj S, Marott JL, et al. Variability of monthly nitrogen multiple-breath washout during one year in children with cystic fibrosis. Journal of cystic fibrosis : official journal of the European Cystic Fibrosis Society. 2018;17(2):242-8.

52. Kirkby J, Bountziouka V, Lum S, Wade A, Stocks J. Natural variability of lung function in young healthy school children. The European respiratory journal. 2016;48(2):411-9.

53. Davies G, Stanojevic S, Raywood E, Duncan JA, Stocks J, Lum S, et al. An observational study of the lung clearance index throughout childhood in cystic fibrosis: Early years matter. The European respiratory journal. 2020.

54. Stocks J, Sly PD, Tepper RS, Morgan WJ. Infant Respiratory Function Testing. New York, NY: John Wiley \& sons, Inc; 1996

55. Singer F, Kieninger E, Abbas C, Yammine S, Fuchs O, Proietti E, et al. Practicability of nitrogen multiple-breath washout measurements in a pediatric cystic fibrosis outpatient setting. Pediatric pulmonology. 2013;48(8):739-46.

56. Bell AS, Lawrence PJ, Singh D, Horsley A. Feasibility and challenges of using multiple breath washout in COPD. Int J Chron Obstruct Pulmon Dis. 2018;13:2113-9.

57. Subbarao P, Milla C, Aurora P, Davies JC, Davis SD, Hall GL, et al. Multiple-Breath Washout as a Lung Function Test in Cystic Fibrosis. A Cystic Fibrosis Foundation Workshop Report. Annals of the American Thoracic Society. 2015;12(6):932-9.

58. Amin R, Subbarao P, Lou W, Jabar A, Balkovec S, Jensen R, et al. The effect of dornase alfa on ventilation inhomogeneity in patients with cystic fibrosis. The European respiratory journal. 2011;37(4):806-12.

59. Shaw M, Khan U, Clancy JP, Donaldson SH, Sagel SD, Rowe SM, et al. Changes in LCl in F508del/F508del patients treated with lumacaftor/ivacaftor: Results from the prospect study. Journal of cystic fibrosis : official journal of the European Cystic Fibrosis Society. 2020.

60. Amin R, Subbarao P, Jabar A, Balkovec S, Jensen R, Kerrigan S, et al. Hypertonic saline improves the $\mathrm{LCl}$ in paediatric patients with CF with normal lung function. Thorax. 2010;65(5):379-83.

61. Stahl M, Wielputz MO, Ricklefs I, Dopfer C, Barth S, Schlegtendal A, et al. Preventive Inhalation of Hypertonic Saline in Infants with Cystic Fibrosis (PRESIS). A Randomized, Double-Blind, Controlled Study. American journal of respiratory and critical care medicine. 2019;199(10):1238-48.

62. Ratjen F, Davis SD, Stanojevic S, Kronmal RA, Hinckley Stukovsky KD, Jorgensen N, et al. Inhaled hypertonic saline in preschool children with cystic fibrosis (SHIP): a multicentre, randomised, doubleblind, placebo-controlled trial. The lancet Respiratory medicine. 2019;7(9):802-9.

63. Saunders C, Jensen R, Robinson PD, Stanojevic S, Klingel M, Short C, et al. Integrating the multiple breath washout test into international multicentre trials. Journal of cystic fibrosis : official journal of the European Cystic Fibrosis Society. 2020;19(4):602-7. 
64. Bayfield KJ, Horsley A, Alton E, Irving S, Bush A, Davies JC. Simultaneous sulfur hexafluoride and nitrogen multiple-breath washout (MBW) to examine inherent differences in MBW outcomes. ERJ Open Res. 2019;5(4).

65. Poncin W, Singer F, Aubriot AS, Lebecque P. Agreement between multiple-breath nitrogen washout systems in children and adults. Journal of cystic fibrosis : official journal of the European Cystic Fibrosis Society. 2017;16(2):258-66.

66. Nixon PA, Orenstein DM, Kelsey SF, Doershuk CF. The prognostic value of exercise testing in patients with cystic fibrosis. The New England journal of medicine. 1992;327(25):1785-8.

67. Pianosi P, Leblanc J, Almudevar A. Peak oxygen uptake and mortality in children with cystic fibrosis. Thorax. 2005;60(1):50-4.

68. Hebestreit H, Hulzebos EHJ, Schneiderman JE, Karila C, Boas SR, Kriemler S, et al. Cardiopulmonary Exercise Testing Provides Additional Prognostic Information in Cystic Fibrosis. (15354970 (Electronic)).

69. Hebestreit H, Arets HG, Aurora P, Boas S, Cerny F, Hulzebos EH, et al. Statement on Exercise Testing in Cystic Fibrosis. Respiration. 2015;90(4):332-51.

70. Godfrey S, Davies CT, Wozniak E, Barnes CA. Cardio-respiratory response to exercise in normal children. Clin Sci. 1971;40(5):419-31.

71. Gulmans VAM, Van Veldhoven NHMJ, De Meer K, Helders PJM. The six-minute walking test in children with cystic fibrosis: Reliability and validity. Pediatric pulmonology. 1996;22(2):85-9.

72. Selvadurai HC, Cooper PJ, Meyers N, Blimkie CJ, Smith L, Mellis CM, et al. Validation of shuttle tests in children with cystic fibrosis. Pediatric pulmonology. 2003;35(2):133-8.

73. Elkins MR, Dentice RL, Bye PT. Validation of the MST-25: an extension of the modified shuttle test (MST). Journal of Cystic Fibrosis. 2009;8:S70.

74. Schneiderman JE, Wilkes DL, Atenafu EG, Nguyen T, Wells GD, Alarie N, et al. Longitudinal relationship between physical activity and lung health in patients with cystic fibrosis. The European respiratory journal. 2014;43(3):817-23.

75. Jantzen A, Opoku-Pare M, Bieli C, Ruf K, Hebestreit $\mathrm{H}$, Moeller A. Perspective on cystic fibrosis and physical activity: Is there a difference compared to healthy individuals? Pediatric pulmonology. 2016;51(10):1020-30.

76. Nixon PA, Orenstein DM, Kelsey SF. Habitual physical activity in children and adolescents with cystic fibrosis. Medicine \& Science in Sports \& Exercise. 2001;33(1):30-5.

77. Selvadurai HC, Blimkie CJ, Cooper PJ, Mellis CM, Van Asperen PP. Gender differences in habitual activity in children with cystic fibrosis. Archives of disease in childhood. 2004;89:928-33.

78. Roach DJ, Cremillieux Y, Fleck RJ, Brody AS, Serai SD, Szczesniak RD, et al. Ultrashort EchoTime Magnetic Resonance Imaging Is a Sensitive Method for the Evaluation of Early Cystic Fibrosis Lung Disease. Ann Am Thorac Soc. 2016;13(11):1923-31.

79. Dournes G, Grodzki D, Macey J, Girodet PO, Fayon M, Chateil JF, et al. Quiet Submillimeter MR Imaging of the Lung Is Feasible with a PETRA Sequence at 1.5 T. Radiology. 2015;276(1):258-65.

80. Marshall H, Horsley A, Taylor CJ, Smith L, Hughes D, Horn FC, et al. Detection of early subclinical lung disease in children with cystic fibrosis by lung ventilation imaging with hyperpolarised gas MRI. Thorax. 2017;72(8):760-2.

81. Wielputz MO, Puderbach M, Kopp-Schneider A, Stahl M, Fritzsching E, Sommerburg O, et al. Magnetic resonance imaging detects changes in structure and perfusion, and response to therapy in early cystic fibrosis lung disease. American journal of respiratory and critical care medicine. 2014;189(8):956-65.

82. Wielputz $\mathrm{MO}$, von Stackelberg $\mathrm{O}$, Stahl M, Jobst BJ, Eichinger $\mathrm{M}$, Puderbach $\mathrm{MU}$, et al. Multicentre standardisation of chest MRI as radiation-free outcome measure of lung disease in young children with cystic fibrosis. Journal of cystic fibrosis : official journal of the European Cystic Fibrosis Society. 2018;17(4):518-27.

83. Woods JC, Wild JM, Wielputz MO, Clancy JP, Hatabu H, Kauczor HU, et al. Current state of the art MRI for the longitudinal assessment of cystic fibrosis. J Magn Reson Imaging. 2019. 
84. Smith LJ, Collier GJ, Marshall H, Hughes PJC, Biancardi AM, Wildman M, et al. Patterns of regional lung physiology in cystic fibrosis using ventilation magnetic resonance imaging and multiplebreath washout. The European respiratory journal. 2018;52(5).

85. Altes TA, Johnson $M$, Fidler $M$, Botfield $M$, Tustison $N J$, Leiva-Salinas $C$, et al. Use of hyperpolarized helium-3 MRI to assess response to ivacaftor treatment in patients with cystic fibrosis. Journal of cystic fibrosis : official journal of the European Cystic Fibrosis Society. 2017;16(2):267-74.

86. Walkup LL, Thomen RP, Akinyi TG, Watters E, Ruppert K, Clancy JP, et al. Feasibility, tolerability and safety of pediatric hyperpolarized (129)Xe magnetic resonance imaging in healthy volunteers and children with cystic fibrosis. Pediatr Radiol. 2016;46(12):1651-62.

87. Rayment JH, Couch MJ, McDonald N, Kanhere N, Manson D, Santyr G, et al. Hyperpolarised (129)Xe magnetic resonance imaging to monitor treatment response in children with cystic fibrosis. The European respiratory journal. 2019;53(5).

88. Smith L, Horsley A, Bray J, Hughes PJ, Biancardi AM, Norquay G, et al. The assessment of short and long term changes in lung function in CF using 129Xe MRI. Eur Respir J. 2020.

89. Martini K, Gygax CM, Benden C, Morgan AR, Parker GJM, Frauenfelder T. Volumetric dynamic oxygen-enhanced MRI (OE-MRI): comparison with CT Brody score and lung function in cystic fibrosis patients. Eur Radiol. 2018;28(10):4037-47.

90. Wielputz MO, Eichinger M, Biederer J, Wege S, Stahl M, Sommerburg O, et al. Imaging of Cystic Fibrosis Lung Disease and Clinical Interpretation. Rofo. 2016;188(9):834-45.

91. Zachariah P, Ryan C, Nadimpalli S, Coscia G, Kolb M, Smith H, et al. Culture-Independent Analysis of Pediatric Bronchoalveolar Lavage Specimens. Ann Am Thorac Soc. 2018;15(9):1047-56.

92. Ahmed B, Bush A, Davies JC. How to use: bacterial cultures in diagnosing lower respiratory tract infections in cystic fibrosis. Archives of disease in childhood Education and practice edition. 2014;99(5):181-7.

93. Stafler $\mathrm{P}$, Mussaffi $\mathrm{H}$, Gendler $\mathrm{Y}$, Blau $\mathrm{H}$. Induced sputum versus broncho-alveolar lavage for pathogen surveillance in young cystic fibrosis patients: Low specificity is not necessarily a bad thing. Pediatric pulmonology. 2018;53(1):8.

94. Quittner AL, Buu A, Messer MA, Modi AC, Watrous M. Development and Validation of the Cystic Fibrosis Questionnaire in the United States: A Health-Related Quality-of-Life Measure for Cystic Fibrosis. Chest. 2005;128(4):2347-54.

95. Alpern AN, Brumback LC, Ratjen F, Rosenfeld M, Davis SD, Quittner AL. Initial evaluation of the Parent Cystic Fibrosis Questionnaire-Revised (CFQ-R) in infants and young children. Journal of Cystic Fibrosis. 2015;14(3):403-11.

96. Rosenfeld M, Ratjen F, Brumback L, Daniel S, Rowbotham R, McNamara S, et al. Inhaled hypertonic saline in infants and children younger than 6 years with cystic fibrosis: the ISIS randomized controlled trial. Jama. 2012;307(21):2269-77.

97. Perrem L, Stanojevic S, Shaw M, Davis S, Retsch-Bogart G, Ratjen F. Changes in the parent cystic fibrosis questionnaire-revised (CFQ-R) with respiratory symptoms in preschool children with cystic fibrosis. Journal of Cystic Fibrosis. 2020;19(3):492-8.

98. Davies J, Sheridan H, Bell N, Cunningham S, Davis SD, Elborn JS, et al. Assessment of clinical response to ivacaftor with lung clearance index in cystic fibrosis patients with a G551D-CFTR mutation and preserved spirometry: a randomised controlled trial. The lancet Respiratory medicine. 2013;1(8):630-8.

99. De Boeck K, Lee T, Amaral M, Drevinek P, Elborn JS, Fajac I, et al. Cystic fibrosis drug trial design in the era of CFTR modulators associated with substantial clinical benefit: stakeholders' consensus view. Journal of cystic fibrosis : official journal of the European Cystic Fibrosis Society. 2020.

100. Rowbotham NJ, Smith S, Leighton PA, Rayner OC, Gathercole K, Elliott ZC, et al. The top 10 research priorities in cystic fibrosis developed by a partnership between people with $\mathrm{CF}$ and healthcare providers. Thorax. 2018;73(4):388-90. 
101. Davies G, Rowbotham NJ, Smith S, Elliot ZC, Gathercole K, Rayner O, et al. Characterising burden of treatment in cystic fibrosis to identify priority areas for clinical trials. Journal of cystic fibrosis : official journal of the European Cystic Fibrosis Society. 2020;19(3):499-502. 